\title{
Optical Performance of the BICEP2 Telescope at the South Pole
}

Randol W. Aikin ${ }^{a}$, P. A. Ade ${ }^{b}$, S. Benton ${ }^{c}$ J. J. Bock ${ }^{a, d}$, J. A. Bonetti ${ }^{d}$, J. A. Brevik ${ }^{a}$, C. D. Dowell $^{a, d}$, L. Duband ${ }^{e}$, J. P. Filippini ${ }^{a}$, S. R. Golwala ${ }^{a}$, M. Halpern ${ }^{f}$, V. V. Hristov ${ }^{a}$, K. Irwin ${ }^{g}$ , J. P. Kaufman ${ }^{h}$, B. G. Keating ${ }^{h}$. J. M. Kovac ${ }^{i}$, C. L. Kuo ${ }^{j, k}$, A. E. Lange ${ }^{a}$, C. B. Netterfield ${ }^{c}$, H. T. Nguyen ${ }^{a}$, R. W. Ogburn IV ${ }^{j, k}$, A. Orlando ${ }^{a}$, C. Pryke ${ }^{l, m}$, S. Richter ${ }^{a}$, J. E. Ruhl ${ }^{n}$, M. C. $^{2}$ Runyan $^{a}$, C. Sheehy ${ }^{l, m}$, S. A. Stokes ${ }^{j}$, R. Sudiwala ${ }^{b}$, G. P. Teply ${ }^{a}$, J. E. Tolan ${ }^{j}$, A. D. Turner ${ }^{d}$, P. Wilson ${ }^{d}$, and C. L. Wong ${ }^{i}$

\author{
${ }^{a}$ California Institute of Technology, 1200 E. California Blvd., Pasadena, CA 91125 USA \\ ${ }^{b}$ School of Physics and Astronomy, Cardiff University, The Parade, Cardiff, CF24 3AA, UK \\ ${ }^{c}$ University of Toronto, Canada \\ ${ }^{d}$ Jet Propulsion Laboratory, 4800 Oak Grove Dr., Pasadena, CA 91109, USA \\ ${ }^{e}$ Commissarait á l'Energie Atomique, Grenoble, France

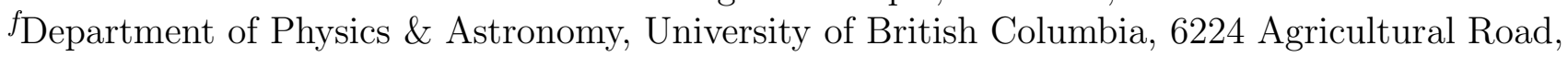 \\ Vancouver, BC V6T1Z1, Canada \\ ${ }^{9}$ NIST Quantum Devices Group, 325 Broadway, Boulder, CO 80305, USA \\ ${ }^{h}$ University of California, San Diego, La Jolla, CA 92093, USA \\ ${ }^{i}$ Department of Astronomy and of Physics, Harvard University, Cambridge, MA 02138, USA \\ ${ }^{j}$ Stanford University, Stanford, 382 Via Pueblo Mall, CA 94305, USA \\ ${ }^{k}$ Kavli Institute for Particle Astrophysics and Cosmology (KIPAC), Sand Hill Road 2575, \\ Menlo Park, CA 94025, USA \\ ${ }^{l}$ University of Chicago, Chicago, IL 60637, USA \\ ${ }^{m}$ University of Minnesota, Minneapolis, MN 55455, USA \\ ${ }^{n}$ Physics Department, Case Western Reserve University, Cleveland, OH 44106, USA
}

\begin{abstract}
BICEP2 deployed to the South Pole during the 2009-2010 austral summer, and is now mapping the polarization of the cosmic microwave background (CMB), searching for evidence of inflationary cosmology. BICEP2 belongs to a new class of telescopes including the KECK Array (ground-based) and SPIDER (balloon-borne) that follow on BICEP's strategy of employing small, cold, on-axis refracting optics. This common design provides key advantages ideal for targeting the polarization signature from inflation, including: (i) A large field of view, allowing substantial light collecting power despite the small aperture, while still resolving the degree-scale polarization of the CMB; (ii) liquid helium-cooled optics and cold stop, allowing for low, stable instrument loading; (iii) the ability to rotate the entire telescope about the boresight; (iv) a baffled primary aperture, reducing sidelobe pickup; and (v) the ability to characterize the far field optical performance of the telescope using ground-based sources. We describe the last of these advantages in detail, including our efforts to measure the main beam shape, beam-match between orthogonally-polarized pairs, polarization efficiency and response angle, sidelobe pickup, and ghost imaging. We do so with ground-based polarized microwave sources mounted in the far field as well as with astronomical calibrators. Ultimately, BICEP2's sensitivity to CMB polarization from inflation will rely on precise calibration of these beam features.
\end{abstract}

Keywords: BICEP, BICEP2, Keck, Spider, Cosmic microwave background, Polarization

Further author information: (Send correspondence to R.W.Aikin)

E-mail: rwa@caltech.edu, Telephone: 16263952016

Millimeter, Submillimeter, and Far-Infrared Detectors and Instrumentation for Astronomy V, edited by Wayne S. Holland, Jonas Zmuidzinas, Proc. of SPIE Vol. 7741, 77410V

(C) 2010 SPIE · CCC code: 0277-786X/10/\$18 - doi: 10.1117/12.857868

Proc. of SPIE Vol. 7741 77410V-1 


\section{INTRODUCTION}

A generic prediction of inflationary cosmology is the presence of a stochastic gravitational wave background. The cosmic microwave background (CMB), the photonic remnant of the epoch of recombination, is predicted to carry a signature from the presence of this gravitational wave background. It has been shown ${ }^{1-3}$ that this signature arises as a curl component in the polarization of the CMB. This is commonly referred to as the $B$-mode component, while the curl-less component is referred to as the $E$-mode component, in analogy with electromagnetism. A number of experiments are now aggressively attempting to measure the $B$-mode power spectrum of the CMB. The current upper limit on the $B$-mode amplitude of the CMB comes from the BICEP telescope ${ }^{4}$ and corresponds to $r<0.7$, where $r$ is the scalar-to-tensor ratio (scalar metric perturbations arising from adiabatic density perturbations, tensor metric perturbations arising from gravitational waves).

To optimize the search, BICEP2 targets the predicted angular and electromagnetic spectral peaks of the primordial $B$-modes. The angular spectrum peaks at scales corresponding to the particle horizon at the time of recombination, which occurs at $\ell \sim 100$. BICEP2 employs the minimum necessary aperture to resolve this peak. The bandcenter $(150 \mathrm{GHz})$ is chosen to coincide with the predicted spectral minimum in foreground contamination (dust + synchrotron emission), while the pass band $(27 \%)$ is chosen to match the well-known atmospheric window at these frequencies.

The observing strategy for BICEP2 closely follows that of BICEP. The instrument is observing from the Dark Sector Laboratory at the South Pole station. Like BiceP, the observing target is the Southern Hole: a region of $800 \mathrm{deg}^{2}$, far from the galactic plane and exceptionally free from galactic dust emission. The field is mapped in a raster pattern, slewing back and forth in azimuth and stepping in elevation. A complete raster of the field is performed every 18 hours at a fixed boresight angle. The field is then mapped at four relative boresight angles $(0,45,180$ and $225 \mathrm{deg})$ for complete $Q$ and $U$ coverage. A complete description of the observing strategy is given in a companion paper in these proceedings. ${ }^{5}$

With dramatically increased mapping speed, BICEP2 aims to improve on BICEP's constraint on $r$. However, increased sensitivity also relies on precise characterization of the optical performance of the telescope. Various beam and polarization systematics (such as pointing offsets between orthogonally polarized detector pairs, polarization orientation uncertainty, etc.) can mix $T$ and $E$-mode signal into $B$-mode signal. For BICEP, these various instrument systematics yield a measurement uncertainty corresponding to $r=0.1{ }^{6}$ This is sufficient for BICEP, but with BICEP2's improved sensitivity, the requirement for careful control of instrument systematics becomes increasingly important.

We describe our efforts to design an optical system well-matched to our observing strategy and detectors, while minimizing polarization contamination. This optical design was deployed to the South Pole and extensively characterized in the 2009-2010 austral summer. This same optical design is also being used in both KECK and SPIDER, both of which will be deploying in the coming year. We summarize our characterization of BICEP2, and discuss our understanding of sources of non-idealities. The goal of this effort is threefold: i) to identify systematic effects to be considered in analysis and simulations; ii) to inform considerations for potential changes to the instrument in our second observing season; and iii) to provide guidance for future CMB polarization missions.

\section{OPTICAL DESIGN}

Before describing the design of the optical system, it is useful to identify the relevant figures of merit. Differential beam parameters are defined as in Takahashi et al. ${ }^{6}$ and can be thought of as a multipole decomposition of the differenced map. The leading terms in this decomposition correspond to beam-width mismatch (monopole), beam center mismatch (dipole) and beam ellipticity mismatch (quadupole). We pursue an optical design that minimizes these differential parameters. In addition to differential beam effects, we consider throughput, optical loading, ghosting, and ground pickup. We optimize in-band transmission and minimize thermal loading in the design of the filter stack and vacuum window. Ghosting is minimized with anti-reflection coatings optimized for the detector band-centers. Finally, ground pickup is minimized by employing a similar baffling scheme to that of BICEP. 


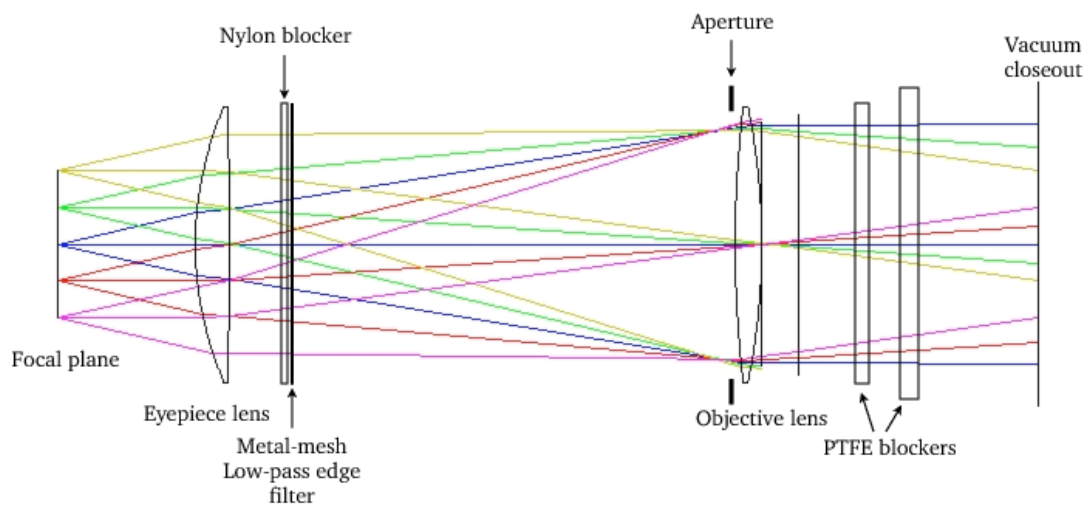

Figure 1. The BICEP2 optical chain.

\subsection{Lens Design}

The lens design was derived from BICEP: a two-lens on-axis refractor. ${ }^{7}$ While more complicated systems (e.g. three lens designs) can yield lower aberration in the focal plane, increasing the number of reflective surfaces introduces polarization-dependent beam "ghosts", and thus proves less attractive for CMB polarimetry. The lenses are high-density polyethylene, cooled to $4.2 \mathrm{~K}$. The aperture stop is chosen to be coincident with the first optic, making the system telecentric. The stop is also cooled to $4.2 \mathrm{~K}$, providing low and stable optical loading. With the BICEP design as a baseline, we further optimize the system for BICEP2.

Before the lens curvature can be fully constrained, the positions of the optical elements must be defined. There are several constraints to consider: The plate scale should yield a spatial resolution corresponding to roughly twice the physical separation of pixels on the focal plane. At these frequencies this requires a focal length $\sim 500$ $\mathrm{mm}$. Constraining the lens separation more precisely requires aberration and diffraction calculations using Zemax simulation software*. Empirically, we find that for $f<550 \mathrm{~mm}$, aberration in corner pixels becomes significant, and results in increased beam ellipticity in the far field. For $f>550 \mathrm{~mm}$, higher aperture illumination, together with the fact that the system cannot be made perfectly telecentric, leads to an increasingly asymmetric illumination of the aperture, and a corresponding increase in beam distortion in the far field. Simulations show that differential polarization effects are not a strong function of the lens separation. The distance from the focal plane to the first optic (eyepiece lens) is chosen to match the beams of the planar phased antennas used in the focal plane. The lens diameters are constrained by the practical consideration of the available space within the cryostat.

With the lens positions defined, the lens curvature is optimized using Zemax modeling. The free parameters include the lens curvature $c$ (reciprocal of the radius of curvature) and conic section $k$ at each surface. The z-coordinate or "sag" as a function of the radial coordinate of the lens is defined in the usual way:

$$
z=\frac{c r^{2}}{1+\sqrt{1-(1+k) c^{2} r^{2}}}
$$

For a telecentric system in the thin-lens limit, the wavefront in the aperture plane is a function of the eyepiece lens curvature only, while the wavefront in the focal plane is a function of the objective lens curvature only. As such, we solve for the eyepiece and the objective lens free parameters separately by considering the illumination of the aperture plane and the focal plane, respectively. We construct two separate models: The first in the time-forward direction, defining 5 collimated ray bundles with an angle of incidence from $0^{\circ}$ to $8^{\circ}$ (corresponding to the field of view), weighted according to the pixel distribution in the focal plane. The objective lens curvature is optimized by minimizing the aberration in the focal plane. The second model is in the time reverse direction, again with 5 collimated ray bundles with an angle of incidence of $0^{\circ}$ to $10^{\circ}$, each weighted according to the relative angular field distribution of our detectors. While holding the objective lens parameters fixed, the eyepiece lens

\footnotetext{
*http://www.zemax.com
} 


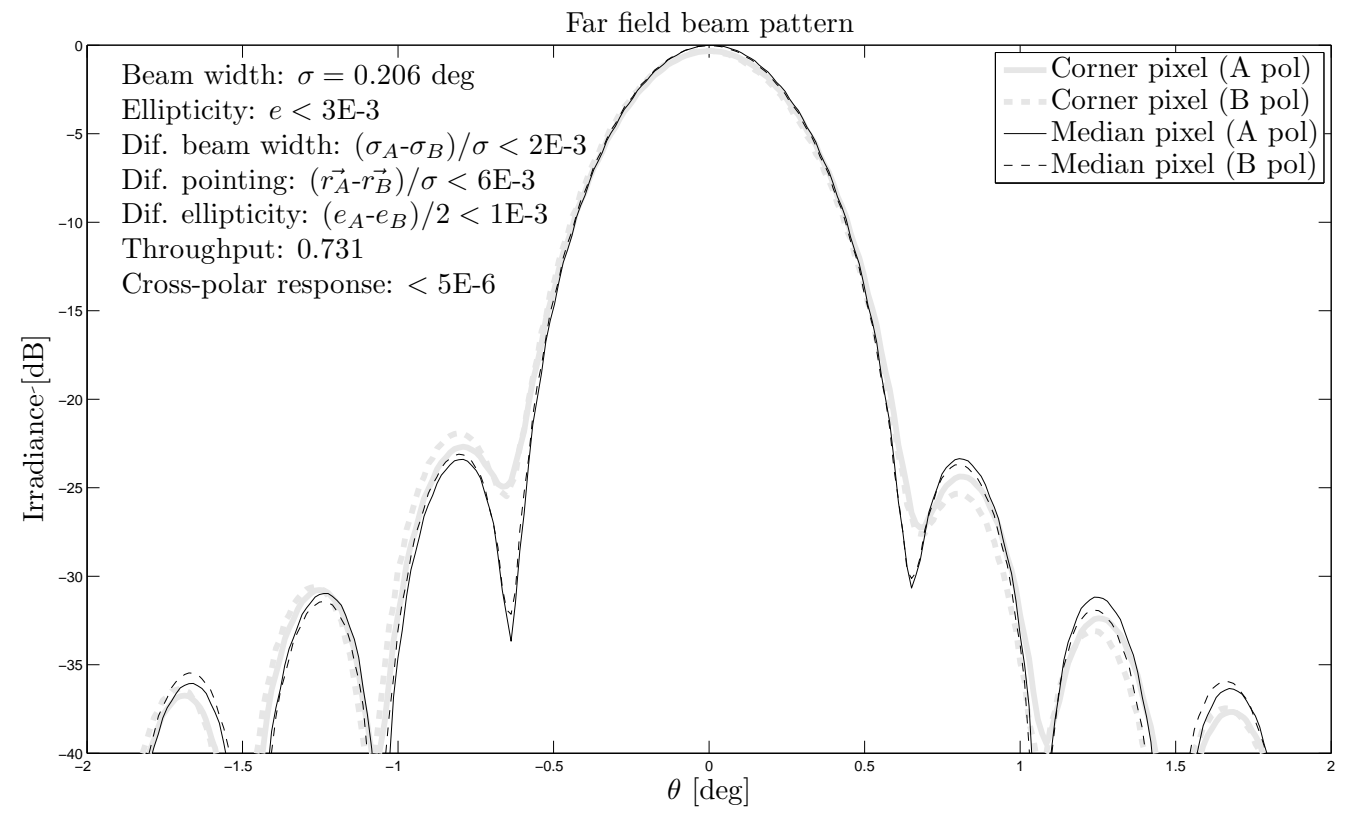

Figure 2. Simulated far field beam pattern for orthogonally polarized A and B beams, for both median and corner pixels. Beam properties are for a median pixel. The median pixel is displaced $56 \mathrm{~mm}$ from the optical axis, and the corner pixel displaced $80 \mathrm{~mm}$, corresponding to the cornermost light pixel in the focal plane. All four share the same normalization. Beam widths, beam centers, and beam ellipticities (and corresponding differential parameters) are upper limits due to the fact that these numerical results become sensitive to the finite sampling used in the simulation at this level. The throughput is calculated with estimated reflection coefficients assigned to all refractive surfaces. With lossless optics, the throughput would be 0.8

is optimized by minimizing the aberration in the aperture plane. Doing so ensures the illumination of the aperture for off-axis pixels is rotationally symmetric. The optimization is performed iteratively by passing the optimization results from the first model into the second, until the free parameters converge.

Aberration in the focal plane can be further reduced by relaxing the telecentric requirement. In practice, this means allowing the eyepiece curvature to be a free parameter in the time-forward model, and minimizing aberration at the focal plane and solving for both lens curvatures simultaneously. The gains are significant, but at the cost of asymmetric illumination of the aperture. Physical optics propagation simulations performed using Zemax predict that requiring telecentricity results in less beam distortion in the far field.

\subsection{Filter stack and vacuum window}

The BICEP2 filter stack consists of two Teflon blockers, a nylon blocker, and a metal mesh filter provided by Peter Ade's group (Fig. 1). The Teflon filters are $30 \mathrm{~mm}$ thick, thermally sunk to the LHe vapor-cooled shields within the cryostat. At these temperatures $(40$ and $100 \mathrm{~K})$ Teflon has excellent in-band transmission. ${ }^{8}$ To further reduce loading, nylon is used between the eyepiece and objective lenses (Fig. 1). The in-band emissivity of nylon is higher than that of Teflon, but substantially cuts the far-IR loading on the sub-Kelvin stages. A metal-mesh low-pass edge filter with a spectral cutoff of $8.3 \mathrm{~cm}^{-1}$ is used in addition to the nylon blocker. This is to mitigate an observed high-frequency spectral leak in the detectors. (Please see reports from Brevik et al. and Orlando et al. in this volume for a complete treatment. ${ }^{9,10}$ ) All of these elements have been anti-reflection coated using a porous PTFE membrane (Mupor ${ }^{\dagger}$ ), with a thickness and porosity chosen to match the impedance of the substrate at $150 \mathrm{GHz}$. The AR coating is heat-bonded using a thin low-density polyethylene film as a

\footnotetext{
${ }^{\dagger}$ http://www.porex.com
} 

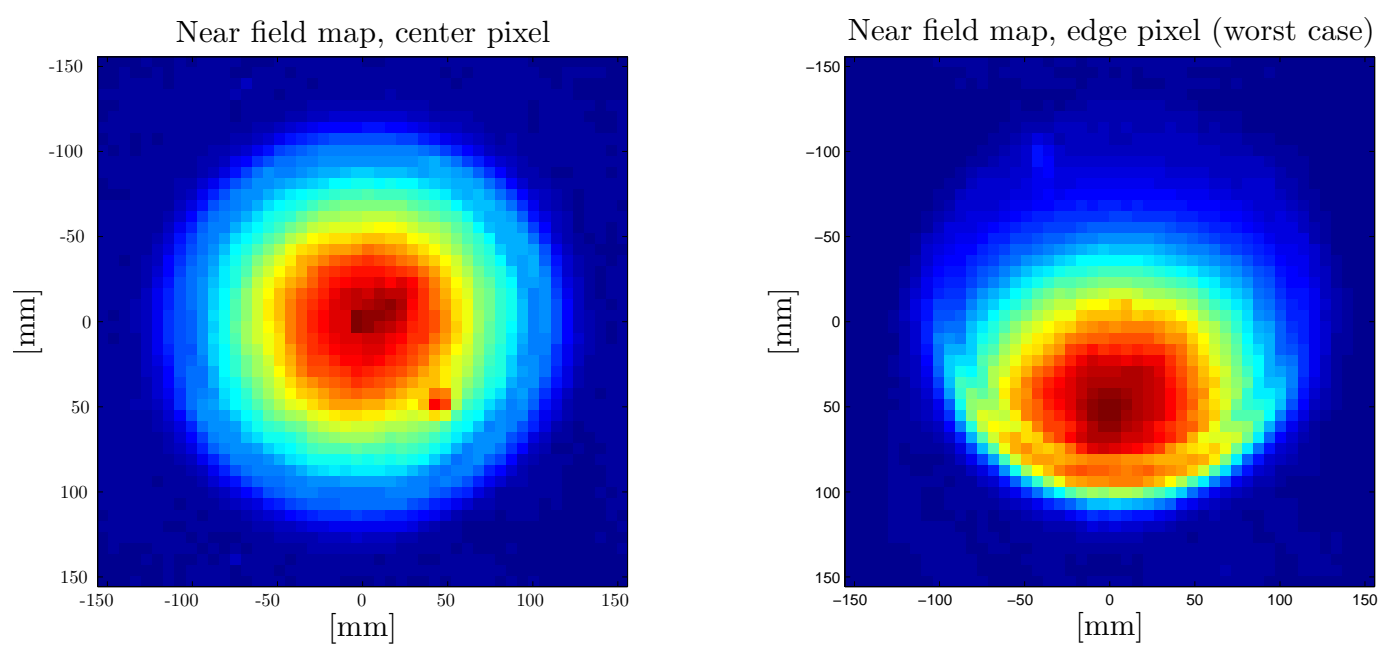

Figure 3. Near field beam maps for a center pixel (typical) and edge pixel (worst case) taken in the plane of the vacuum window. Color scale is linear. The sharp peak in the left figure is caused by ghost reflections off of the $4 \mathrm{~K}$ nylon blocker. The asymmetric illumination of the aperture seen in the edge pixel is believed to be caused by a phase gradient in the phased-array antenna. While roughly $25 \%$ of pixels exhibit this behavior at some level, only about $6 \%$ suffer from the severe asymmetry pictured.

bonding layer and is pressed against the lens surface using a vacuum bag, ensuring uniform pressure across the lens surface.

The vacuum closeout window is Zotefoam PPA30 polypropylene foam ${ }^{\ddagger}$, of the same variety used in BICEP. The transmission of the window was measured using BICEP2 and a chopped thermal source and found to be $>98 \%$. The foam is bonded to an annular aluminum frame. Zemax simulations show that the edge illumination of this frame (as well as the frames supporting the Teflon blockers) is at or below $-40 \mathrm{~dB}$ for corner pixels.

\section{BEAM CHARACTERIZATION}

This optical design was deployed to the South Pole during the 2009-2010 austral summer. In an effort to identify potential sources of systematic uncertainty, the BICEP2 optics were characterized in both the near and far field. Far field maps serve to verify the parameters outlined in Fig. 2, while near field measurements prove to be a powerful tool in diagnosing the optical performance of the detectors themselves.

\subsection{Near field maps}

To probe the optical performance of the detectors, we map the response of the instrument in the plane of the window. Mapping at this position ensures that we are well within the Fresnel region of the diffracting aperture of the optics $(\sqrt{\lambda d}<<D$, where $d$ is the distance to the plane of the map and $D$ is the aperture diameter) and within the Fraunhofer region of the detectors. As a result, we are highly sensitive to the phase distribution of the field in the plane of the detectors, but (to first approximation) insensitive to the phase distribution of the field in the plane of the aperture. The map is made using a two-stage linear actuator mounted directly above the window of the telescope. The source is a radiative heater, coated with carbon-loaded silicone rubber for blackness. A typical near field map is plotted in Fig. 3 (left). A ghost reflection off of the nylon filter back to the focal plane is seen as a sharp peak in the plane of the map, and has an integrated power of $10^{-4}$ as compared to the main beam.

The most striking distortion appears in edge pixels, which clearly exhibit an asymmetric illumination of the aperture as seen in Fig. 3 (right). We refer to this as a "beam-steer" effect, and understand it to arise from

\footnotetext{
${ }^{\ddagger}$ http://http://www.zotefoams.com
} 

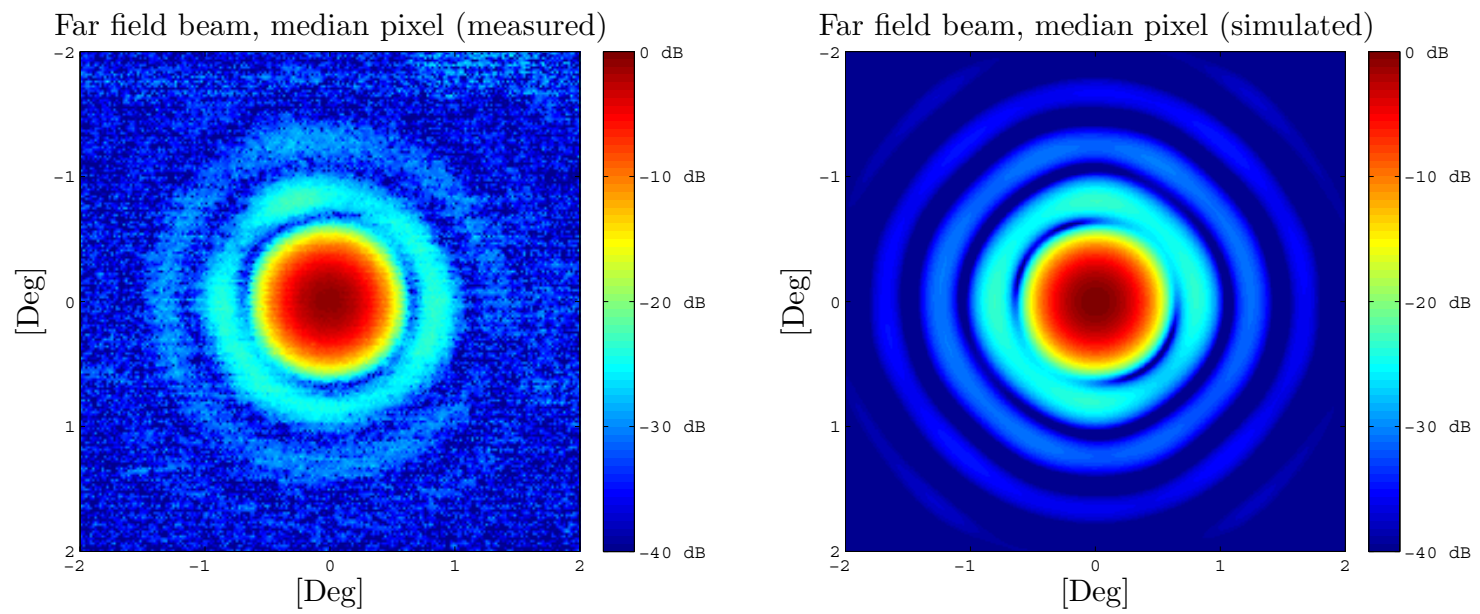

Figure 4. Left: Far field beam map for a pixel, median in its displacement from the optical axis, measured with a broadband microwave source. Right: Simulated beam pattern for the same displacement from the optical axis. Color scale is logarithmic.

a phase gradient in the field distribution in the plane of the detectors. Physical optics propagation simulations are in strong agreement with this hypothesis. We find that the phase gradient in the focal plane required to produce the observed beam pattern is $180^{\circ}$ or larger across a pixel. A physical tilt of the pixel is not plausible, and the thickness variation in the inter-layer dielectric (ILD) of the antenna has been measured to be too small to explain the effect. However, this can result from a variation in the ILD index of refraction. The variation in index required to produce a phase gradient of $180^{\circ}$ (corresponding to an optical path length difference of $\lambda / 2$ ) is $\sim 0.1$, or a fractional variation of $\sim 5 \%$ across a pixel. We are now pursuing this as a likely explanation, and actively testing new devices to probe this index variation.

There are several consequences associated with this effect. Obviously, highly steered pixels are poorly coupled to the sky. In addition, increased far field beam ellipticity results from the asymmetric beam truncation by the asymmetrically illuminated aperture. For most pixels, beam mismatch in the far field is not dominated by this beam-steering effect. However, we do find that polarization pairs suffering from severe beam-steer are poorly matched in the far field. This tends to result in peculiar differential beam distortion, rather than a simple pointing offset or a beam-width mismatch.

\subsection{Far field maps}

While near field maps are a useful diagnostic tool, ultimately it is the far field beam pattern that determines the degree to which beam systematics introduce measurement uncertainty in $r$. We have mapped the far field beam distribution using both the Moon and an amplified microwave noise source. The microwave source was attached to a mast on the Martin A. Pomerantz Observatory (MAPO) building at a range of $200 \mathrm{~m}$. The microwave source has a bandwidth of $\sim 15 \%$ centered at $150 \mathrm{GHz}$ and can be coupled to either a linear or circular polarizer. To direct the telescope's beam to the source, a flat mirror was mounted above the telescope.

The main beam for a typical pixel in the far field is shown in Fig. 4. Main beam features are generally found to be in strong agreement with Zemax physical optics propagation simulations. However, some pixels exhibit higher beam ellipticity than predicted. This is believed to originate from the asymmetric aperture illumination described in Sec. 3.1. In addition to the primary beam features pictured, wider scans reveal ghost images that have an integrated power of $\sim 10^{-4}$, roughly an order of magnitude improvement over BICEP1. There are also "crosstalk beams" - secondary beams that result from inductive crosstalk in the SQUID multiplexing system. The amplitude is consistent with nearest-neighbor first stage SQUID crosstalk measured previously by NIST, ${ }^{11}$ at or below $3 \times 10^{-3}$. 

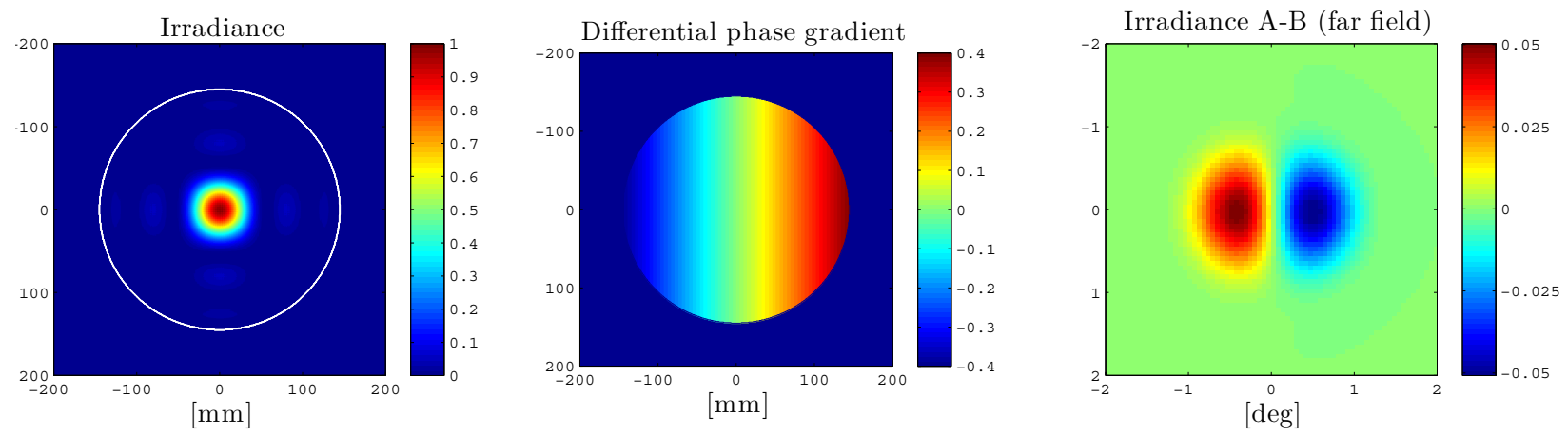

Figure 5. Left: Simulated irradiance pattern for a center pixel, A polarization, in the plane of the eyepiece lens. The white circle indicates the diameter of the lens for comparison. Center: The differential phase gradient in the plane of the eyepiece lens required to produce the observed far field mismatch (in radians). Right: Predicted far field beam mismatch for the differential phase gradient plotted (center). The amplitude corresponds to what is typically observed in the far field with BICEP2.

\section{POLARIZATION CHARACTERIZATION AND DIFFERENTIAL BEAM EFFECTS}

Using the far field mapping technique described above, we are able to investigate the polarization performance of the telescope using linearly and circularly polarized broadband noise sources. We measure differential beam effects and also attempt to place upper limits on the polarization efficiency of the detectors.

\subsection{Differential maps}

A variety of differential beam effects can lead to temperature to polarization leakage, as described in Sec. 1. The most prominent effect observed is a shift in the beam centers of orthogonally polarized A and B pixels. This has been measured using both far field microwave sources as well as Moon observations. A similar effect was observed in BICEP $1,{ }^{6}$ but typical values for the A and B angular beam center displacement are reported to be roughly an order of magnitude lower in BICEP1. In BICEP2, the symmetry of the A-B mismatch leads us to believe the offset is caused by something in the optical chain. In particular, the fact that the direction and amplitude of the pointing offset seem to be tied to focal plane position, rather than tile position, suggests that the origin of the mismatch arises from the optics, rather than the detectors themselves. While a number of possible causes are being actively pursued, the mismatch could be a consequence of a spatial gradient in the birefringence of one of the refracting elements. A spatial gradient in the birefringence of the objective lens or Teflon filters would result in an offset in the far field constant in both amplitude and direction due to the fact that the beams largely overlap on these surfaces. A spatial gradient in the nylon blocker, eyepiece lens, or metal-mesh filter would result in an offset that varies across the focal plane, as we observe.

As in the case of the near-field, we can investigate the phase gradient required to produce the observed far field mismatch. The result is plotted in Fig. 5. By introducing a differential phase gradient in the plane of the nylon filter, we find that a differential linear phase gradient of roughly $2 \times 10^{-3}$ radians $/ \mathrm{mm}$ results in the far field beam mismatch observed. The simulation has been performed in the plane of the nylon filter, but the results in the plane of the eyepiece lens are very similar. To see if this can plausibly be explained by material birefringence, we estimate the required index variation required to produce this phase gradient. The optical path length difference resulting from this phase gradient is $\Delta \phi \lambda /(2 \pi)$, where $\Delta \phi$ is predicted to be $\simeq 0.2$ radians (Fig. 5). Setting this equal to $\Delta n d$, where $d$ is the thickness of the material, we find that $\Delta n / n_{0}$, the fractional index variation, will be on the order of $1 \%$. In other words, a spatially varying birefringence, on the order of $1 \%$, due to perhaps anisotropic stress or material impurities, could substantially effect the beam match in the far field. 


\begin{tabular}{|c|c|c|c|c|}
\hline Parameter & Definition & $\begin{array}{c}\text { BICEP } \\
\text { Measured }^{1}\end{array}$ & Calculated $^{2}$ & $\begin{array}{l}\text { BICEP2 } \\
\text { Preliminary measurement }{ }^{3}\end{array}$ \\
\hline $\begin{array}{l}\text { Beam width } \\
\text { Beam ellipticity } \\
\text { Differential beam width } \\
\text { Differential pointing } \\
\text { Differential ellipticity }\end{array}$ & $\begin{array}{c}\sigma=\alpha \\
e=\left(\sigma_{m a j}-\sigma_{\min }\right) / \sigma \\
\left(\sigma_{1}-\sigma_{2}\right) / \sigma \\
\Delta \theta / \sigma \\
\left(e_{1}-e_{2}\right) / 2\end{array}$ & $\begin{array}{c}0.26 \mathrm{deg} \\
<0.01 \\
<3 \times 10^{-3} \\
1.3 \times 10^{-2} \\
<2 \times 10^{-3}\end{array}$ & $\begin{array}{l}0.201 \text { Deg } \\
<3 \times 10^{-3} \\
<7 \times 10^{-3} \\
<6 \times 10^{-3} \\
<1 \times 10^{-3}\end{array}$ & $\begin{array}{c}0.221 \pm 0.006 \\
0.03 \pm 0.03 \\
0.003 \pm 0.002 \\
0.1 \pm 0.04 \\
0.012 \pm 0.09\end{array}$ \\
\hline
\end{tabular}

Table 1. Beam properties, measured and calculated, for BICEP and BICEP2.

\subsection{Polarization efficiency}

In addition to differential beam properties, polarization efficiencies have been measured to be at or below $5 \times 10^{-3}$ using a linearly polarized microwave source in the far field. A substantial fraction of this polarization leakage is due to the inductive nearest-neighbor coupling of first-stage SQUIDs, since A/B polarization pairs are coupled to neighboring first-stage SQUIDs.

\section{CONCLUSIONS}

Constraining $r$ demands both raw sensitivity and careful control of systematics. This requires precise knowledge of the optical performance of the telescope. After extensive testing, we find two sources of potentially large systematic contamination for BICEP2. The first, as described, is the "beam-steer" effect observed in the near field. In addition to increased beam ellipticities and degraded optical efficiency, this can cause differential beam distortion in the far field. There is evidence that this arises from a phase gradient in the plane of the detectors, and could be caused by a spatial variation in the ILD index of the planar phased antennas. The second is the differential pointing observed in the far field between orthogonally polarized detector pairs. As we have discussed, the behavior is consistent with a linear differential phase gradient induced by one or more of the refracting elements of the system.

Without correction, these systematics, in particular the observed differential pointing, could quickly dominate BICEP2's measurement uncertainty in $r$. BICEP1 simulations found that if differential pointing were to average $1.9 \%$, it could give rise to false signal at the level corresponding to $r=0.1{ }^{6}$ While BICEP2 observes a larger offset, there are substantial gains to be had in analysis. We are now investigating new analysis techniques aimed at correcting for differential pointing centers, to suppress the associated systematic uncertainty below the noise floor for the instrument.

In addition to analysis solutions, hardware solutions are also being pursued for BICEP2's second observing season. New devices using a different ILD material are being fabricated to reduce index variation. And while the source of the far field differential pointing is not completely understood, we have evidence to suggest that removing sources of spatially varying birefringence in the optical chain could substantially reduce the effect. An additional hardware solution is a rotating half-wave plate, which is being tested for similar telescopes (KECK and SPIDER) and is also being considered for BICEP2's second season.

A key advantage to using a small aperture, large field of view optical design is the ability to thoroughly characterize the optical performance from the ground by making beam measurements in the near and far field. This has enabled us to identify, diagnose, and respond to potential sources of systematic uncertainty. We have been successful in identifying far field beam non-idealities and polarization-pair beam mismatch, which we can combat in hardware and analysis. Additionally, near field tests have proven to be a powerful tool in evaluating the optical performance of the phased array antennas. In the end, this precise knowledge of the optical performance of the telescope will lead to an increased sensitivity to the $B$-mode component of the cosmic microwave background, and thus, the energy scale of inflation. 


\section{ACKNOWLEDGMENTS}

BICEP2 has been made possible by support from the National Science Foundation, Grant No. ANT-0742818. Detector development has been made possible by the generous support of the Gordon and Betty Moore Foundation. We are grateful to have Steffen Richter as our 2010 South Pole winterover. The BICEP2 team would also like to thank the South Pole Station staff for logistical support. We thank our BICEP, KECK, and SPIDER colleagues for useful discussions and shared expertise. Finally we wish to thank Barbara Wertz and Kathy Deniston for their tireless logistical and administrative support.

\section{REFERENCES}

1. Seljak, U., "Measuring Polarization in the Cosmic Microwave Background," Astrophys. J 482 (June 1997).

2. Kamionkowski, M., Kosowsky, A., and Stebbins, A., "A Probe of Primordial Gravity Waves and Vorticity," Physical Review Letters 78 (Mar. 1997).

3. Seljak, U. and Zaldarriaga, M., "Signature of Gravity Waves in the Polarization of the Microwave Background," Physical Review Letters 78 (Mar. 1997).

4. Chiang, H. C., Ade, P. A. R., Barkats, D., Battle, J. O., Bierman, E. M., Bock, J. J., Dowell, C. D., Duband, L., Hivon, E. F., Holzapfel, W. L., Hristov, V. V., Jones, W. C., Keating, B. G., Kovac, J. M., Kuo, C. L., Lange, A. E., Leitch, E. M., Mason, P. V., Matsumura, T., Nguyen, H. T., Ponthieu, N., Pryke, C., Richter, S., Rocha, G., Sheehy, C., Takahashi, Y. D., Tolan, J. E., and Yoon, K. W., "Measurement of Cosmic Microwave Background Polarization Power Spectra from Two Years of BICEP Data," Astrophys. J. 711, 1123-1140 (2010).

5. Ogburn, R. W. et al., "The BICEP2 CMB polarization experiment," These Proceedings (2010).

6. Takahashi, Y. D., Ade, P. A. R., Barkats, D., Battle, J. O., Bierman, E. M., Bock, J. J., Chiang, H. C., Dowell, C. D., Duband, L., Hivon, E. F., Holzapfel, W. L., Hristov, V. V., Keating, B. G., Kovac, J. M., Kuo, C. L., Lange, A. E., Leitch, E. M., Mason, P. V., Nguyen, H. T., Ponthieu, N., Pryke, C., Richter, S., Rocha, G. D., and Yoon, K. W., "Characterization of the bicep telescope for high-precision cosmic microwave background polarimetry," Astrophys. J. 711, 1141-1157 (2010).

7. Yoon, K. W., Ade, P. A. R., Barkats, D., Battle, J. O., Bierman, E. M., Bock, J. J., Brevik, J. A., Chiang, H. C., Crites, A., Dowell, C. D., Duband, L., Griffin, G. S., Hivon, E. F., Holzapfel, W. L., Hristov, V. V., Keating, B. G., Kovac, J. M., Kuo, C. L., Lange, A. E., Leitch, E. M., Mason, P. V., Nguyen, H. T., Ponthieu, N., Takahashi, Y. D., Renbarger, T., Weintraub, L. C., and Woolsey, D., "The robinson gravitational wave background telescope (bicep): a bolometric large angular scale cmb polarimeter," Proceedings of SPIE 6275 (2006).

8. Halpern, M., Gush, H. P., Wishnow, E., and de Cosmo, V., "Far infrared transmission of dielectrics at cryogenic and room temperatures: glass, fluorogold, eccosorb, stycast, and various plastics," Applied Optics 25, 565-570 (1986).

9. Orlando, A. et al., "Antenna-coupled TES bolometer arrays for BICEP2/Keck and SPIDER," These Proceedings (2010).

10. Brevik, J. B. et al., "Initial performance of the BICEP2 antenna-coupled superconducting bolometers at the South Pole," These Proceedings (2010).

11. de Korte, P. A. J., Beyer, J., Deiker, S., Hilton, G. C., Irwin, K. D., Macintosh, M., Nam, S. W., Reintsema, C. D., Vale, L. R., and Huber, M. E., "Time-division superconducting quantum interference device multiplexer for transition-edge sensors," Review of Scientific Instruments 74 (Aug. 2003). 